\title{
Mechanical dysfunction in the mucosal oedema formation of patients with nasal polyps*
}

\author{
Rogério Pezato ${ }^{1,2}$, Richard Louis Voegels ${ }^{3}$, Thiago Freire Pinto Bezerra ${ }^{3}$, \\ Claudina Perez-Novo', Aldo Cassol Stamm², Luís Carlos Gregório² \\ 1 Upper Airway Research Laboratory (URL), Department of Otorhinolaryngology, Ghent University Hospital, \\ Ghent University, Belgium \\ 2 Department of Otolaryngology-Head and Neck Surgery, Federal University of São Paulo, Brazil \\ ${ }^{3}$ Department of Otorhinolaryngology and Ophthalmology, University of São Paulo, Brazil
}

Rhinology 52: 162-166, 2014

DOI:10.4193/Rhino13.066

*Received for publication:

May 18, 2013

Accepted: December 22, 2013

\begin{abstract}
Background: Nasal polyposis (NP) is characterized by an anomalous tissue growth with oedema and a lack of extracellular matrix. In this study, we investigated whether a mechanical disfunction of the forces that act in oedema formation is present in NP.

Methods: We compared the interstitial hydrostatic pressure behaviour during a saline solution infusion between healthy nasal mucosa (inferior and middle turbinate from 10 patients) and inflamed nasal mucosa from NP patients (inferior, middle turbinate and a nasal polyp from 6 patients). We used Controlled Disc Stimulation equipment to compare the curve Pressure/Volume created during the saline solution infusion.
\end{abstract}

Results: The pressure at $0.2 \mathrm{ml}$ infusion was lower in the middle turbinate of NP patients than in the middle turbinate of control patients. The lowest P/V mean assessed was in the polypoid tissue.

Conclusions: The interstitial hydrostatic pressure showed different behaviour during liquid infusion in nasal mucosa from NP patients when compared with healthy nasal mucosa. This study allows us to cogitate on a new pathophysiological mechanism contributing to the development of the NP.

Key words: nasal polyposis, polyp, mucosa, chronic sinusitis, hydrostatic pressure

\section{Introduction}

Nasal polyposis (NP) is a chronic sinus disease with a prevalence estimated to be as high as $4 \%$ in the general population. It often occurs in association with severe asthma, aspirin intolerance and cystic fibrosis ${ }^{(1)}$.

The majority of studies in NP are concentrated on understanding the underlying inflammatory process and the exposures that provoke it such as fungus (2), staphylococcus enterotoxins ${ }^{(3)}$, allergens and pollution ${ }^{(4)}$. A strong genetic marker and an effective animal model are lacking, factors that limit the study of NP. Eosinophils were long considered the main cells found in the nasal polyp, with the inflammatory process being orchestrated by Th2 cells. This paradigm has been changed by studies in Asian populations that found a predominance of neutrophils and a high influence of Th1/Th17 cells ${ }^{(5)}$.

It is intriguing that the upper and lower airway share the same pseudostratified ciliated columnar epithelium lining. Many consider this epithelium as a continuum, constituting a single airway, being affected by similar inflammatory processes in several disease states, for example asthma and nasal polyposis. These diseases share hallmarks: predominant infiltration of eosinophils, mucus cell hyperplasia, oedema, thickened basal membrane, a Th2 drived immune response, and increased pro-inflammatory mediators (e.g. cysteinyl leukotrienes) ${ }^{(6,7)}$. Although there is similarity in the histology and inflammatory process in the nasal and bronchial mucosa, this is not true for 
polyposis, which is never found in the bronchial mucosa. Instead of being a specific inflammatory process that characterizes NP, it could rather be a severe inflammation, initiated by chemical or biological agents, that trigger tissue hypertrophy in susceptible nasal mucosa.

Studies involving extracellular matrix in NP have demonstrated decreased TGF-beta production and a lack of collagen in nasal polyps when compared to those in healthy subjects ${ }^{(8)}$. We have confirmed these findings showing a decreased expression of TGF-beta in the submucosa of NP patients ${ }^{(9)}$.

As there is no standard inflammatory process for NP as cited above, the lack of TGF-beta expression and extracellular matrix in the NP submucosa, and the similar inflammatory process of the pseudostratified ciliated columnar epithelium lining causes polyposis in the nasal mucosa but not in the bronchial mucosa led us to hypothesize that the nasal mucosa of patients with NP offers less resistance to oedema formation during the inflammatory process ${ }^{(10)}$.

To address our hypothesis, we studied the mechanical forces involved in oedema formation. The assessment of interstitial fluid pressure has been widely used in oncology, using a sharpened glass capillary connected to a counter-pressure system or a fine steel needle filled with a wick connected to a counter-pressure device (wick-in-needle technique) (11).

In this study, we evaluated the increase of interstitial pressure during saline infusion in the nasal mucosa of NP patients and healthy subjects.

\section{Materials and methods}

\section{Study design}

Patients (Table 1) from the department of Otorhinolaryngology, Federal University of São Paulo, Brazil, were selected and included between August 2012 and January 2013. Inferior and middle turbinates from 10 septoplasty patients without sinus disease, and inferior, middle turbinates and nasal polyps from 6 NP patients (according to the European Position Paper on Rhinosinusitis and Nasal Polyposis 2012) ${ }^{(12)}$ who underwent functional endoscopic surgery were studied.

The pressure within the submucosa was measured during infusion of 0.2 to $1 \mathrm{ml}$ of saline solution $(0.9 \% \mathrm{NaCl})$. The patients were under general anesthesia, and no intranasal drug was administered before the saline solution injection.

To inject a controlled volume within the submucosa and to measure the local pressure we used the Controlled Disc Stimulation (CDS, Smith \& Niephew, Memphis, TN, USA). For the injection, a 22-gauge needle was connected to the pressure-measuring device.

This study was approved by the local ethics commitee of the Federal University of São Paulo, Brazil, registered as 01510612.8.0000.5505. An informed consent was obtained from each patient before study participation.

\section{Statistical analysis}

Study data were analyzed using SPSS software version 18 (IBM Corporation, NY, USA). The Mann-Whitney $U$ test was applied to evaluate the statistical differences between patient groups. $P$ values of less than 0.05 were regarded as significant.

\section{Results}

There was no difference in age between control (from 21 to 46 years) and NP group (from 14 to 78 years), $(p=0.27)$.

Within each patient group, the presence of rhinitis (in control group $p=0.16$ for inferior turbinate and $p=1$ for middle turbinate, in NP group $p=0.18$ for inferior turbinate and $p=0.31$ for middle turbinate) did not alter the interstitial hydrostatic pressure after $0.2 \mathrm{ml}$ infusion $(\mathrm{p}>0.05)$.

The presence of asthma could not be properly evaluated due the a low prevalence within the groups.

Table 1. Patients: clinical characterization.

\begin{tabular}{llcccc}
\multicolumn{7}{c}{ Demographic characteristics } & & \\
Identification & Gender & Disease & Asthma & Rhinitis & Smoking \\
AMS & female & control & + & + & - \\
MMDS & female & control & - & + & + \\
VLS & female & control & - & + & - \\
VAT & male & control & - & - & - \\
EMC & male & control & - & - & - \\
FCP & male & control & - & + & - \\
FFDM & male & control & - & - & - \\
EA & female & control & - & - & - \\
POR & female & control & - & + & - \\
ERO & male & control & - & - & - \\
AO & male & NP & - & - & - \\
OBS & male & NP & - & - & - \\
MAO & male & NP & - & + & + \\
EABS & male & NP & - & + & - \\
CRC & male & $\mathrm{NP}$ & + & + & - \\
JSS & male & $\mathrm{NP}$ & + & + & - \\
\hline & & & + & + \\
\hline
\end{tabular}



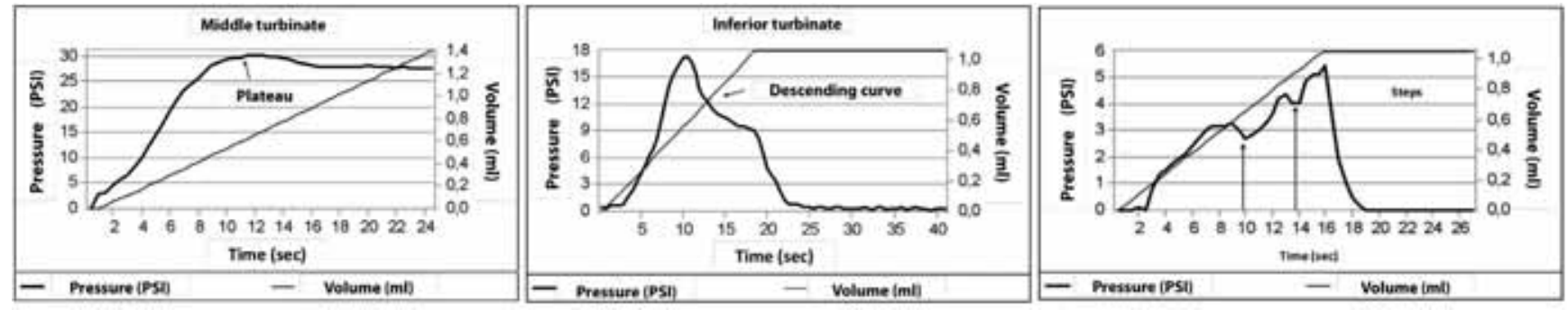

Figure 1. Demonstration of leaking saline solution: (A) plateau formation, (B) descending curve, (C) presence of steps.

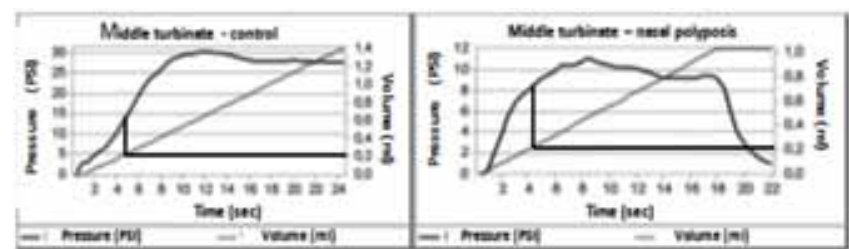

A

Figure 2. Illustration comparing peak of pressure at $0.2 \mathrm{ml}$ of infusion. Pressure in healthy nasal mucosa (A) was higher (13 PSI) than in nasal mucosa from patient suffering of NP (B, 8 PSI).

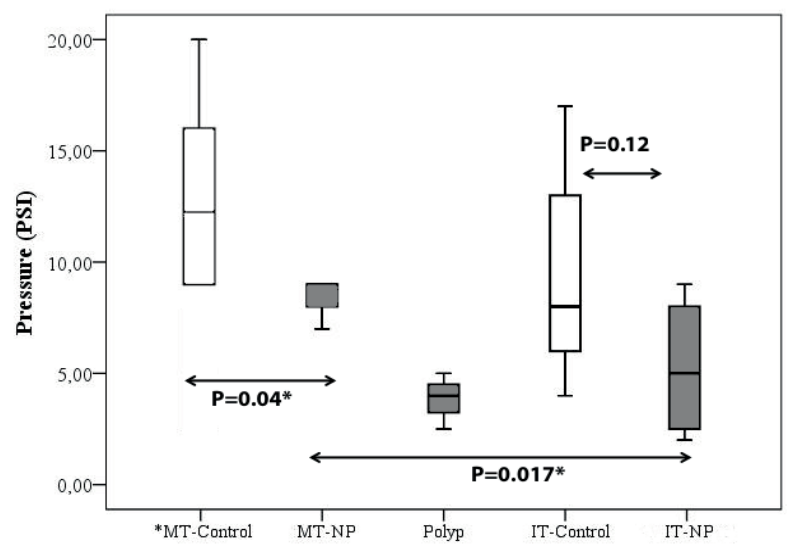

Figure 3. The pressure in the middle turbinate from healthy nasal mucosa is higher after $0.2 \mathrm{ml}$ infusion when compared with middle turbinate from NP nasal mucosa. The polypoid tissue reaches the lowest pressure after $0.2 \mathrm{ml}$ of infusion followed by inferior turbinate. The middle turbinate reaches the highest pressure.

The endoscopic visualization of saline solution extravasation into the nasal cavity was illustrated by a plateau or descending curve (Figure 1) in a graph of pressure versus volume.

\section{Pressure reached at $0.2 \mathrm{ml}$ infusion.}

The patient graphs that showed a plateau, a descending curve or a step (notch in the graph) before reaching the $0.2 \mathrm{ml}$ infusion volume were not included in the analysis ( 2 inferior turbinates from the control group and 1 from the NP patient goup, 3

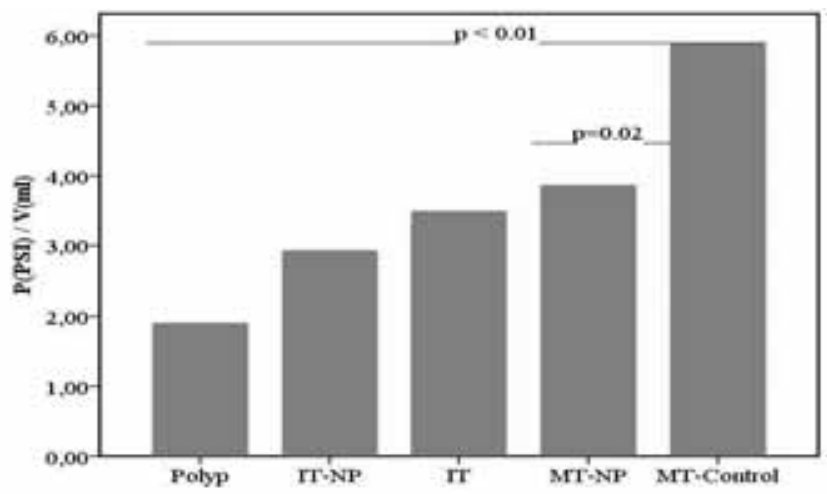

Figure 4. Illustration of the increase of pressure per $0.1 \mathrm{ml}$ added into the nasal mucosa. The highest increase was found in middle turbinate and the lowest in polyps. The increase of pressure was lower in samples with a diagnosis of NP.

middle turbinates from controls and 1 from NP patients, and 2 polyps). The pressure reached at $0.2 \mathrm{ml}$ infusion had a tendency to be lower, but it was not significantly different in the inferior turbinate from patients with NP when compared with the inferior turbinate of healthy patients $(p=0.12)$. For the middle turbinate, NP patients had a lower pressure at $0.2 \mathrm{ml}$ infusion when compared to healthy patients ( $p=0.04$ )(Figures 2 and 3 ). We observed a tendency for the pressure to be lower at $0.2 \mathrm{ml}$ volume in the submucosa of inferior turbinates than of middle turbinates. Polypoid tissue had the lowest pressure of all assessed tissues (Figure 3).

Increase of pressure versus volume (PSI/ml)

The first part of the pressure-volume (P/V) graph demonstrated linear variation; we therefore used the $\mathrm{P} / \mathrm{V}$ mean to compare the hydrostatic pressure created during infusion. No graph was excluded from the analysis.

We observed that the P/V mean was lower in the submucosa of inferior turbinates than in the submucosa of middle turbinates, regardless of the diagnosis. Considering the turbinate, we observed that the P/V mean was lower in the NP group when compared to the control group. Polypoid tissue had the lowest $P / V$ mean of all. The P/V mean was significantly lower in polypoid 


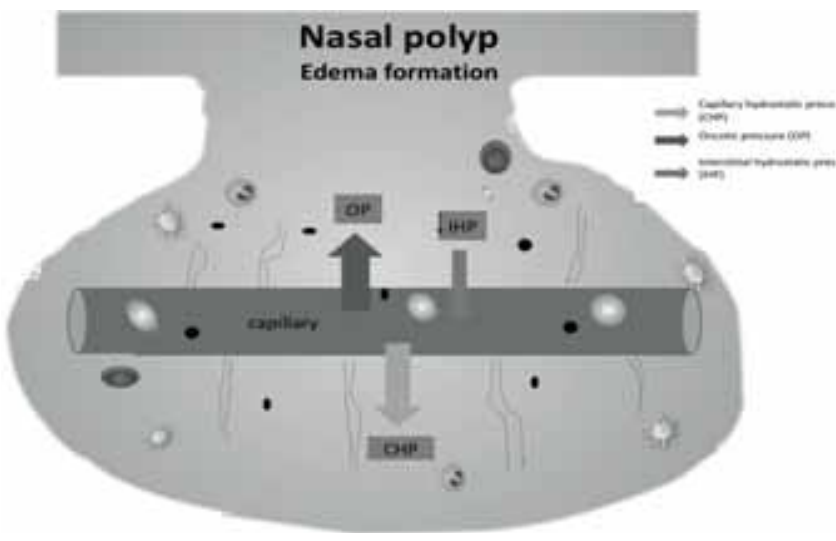

Figure 5. Scheme showing possible mechanisms to oedema formation in NP. During the inflammatory process, the interstitial hydrostatic pressure (IHP) is lower in NP than in healthy nasal mucosa, consequently IHP is not able to counterbalance the increase of interstitial osmotic pressure (IOP) found due the protein extravasation from the capillary (exudate).

tissue compared to middle turbinates from healthy volunteers $(\mathrm{p}<0.01)$ (Figure 4).

\section{Discussion}

We have demonstrated that there are mechanical differences in the behaviour of interstitial pressure during infusion of fluid between healthy nasal mucosa and nasal mucosa from patients with NP (Figure 5).

The increase of interstitial hydrostatic pressure in normal nasal mucosa, when compared to nasal mucosa from NP patients, could partially counterbalance the decrease of capillary oncotic pressure (capillary osmotic pressure - interstitial osmotic pressure) during the inflammatory process (where there is extravasation of protein and consequently water). This could serve to prevent extravasation of water from the vessel to the interstium and therefore minimize oedema formation.

In solid tumours, it has been demonstrated that an increase in the interstitial fluid pressure hinders therapeutic drugs to reach neoplastic cells which has been identified as an important barrier to drug delivery ${ }^{(11,13)}$. High fluid pressure is associated with increase of matrix stiffness and increased interstitial flow in tumours ${ }^{(14)}$.

In parallel, the lower pressure found in the NP mucosa when compared with healthy mucosa after acute infusion of a volume in this study matches with the lack of extracellular matrix and TGF-beta ${ }^{(8,9)}$ found in NP mucosa. The technique and equipment applied in this study follow the concepts used to evaluate interstitial fluid pressure within tumour tissue added the possibility of injection of solution during the measurement of pressure (11). Some samples could not be included due to leakage of solution from the submucosa into the nasal cavity, indicating the need for development of more sensitive equipment able to detect variations in pressure with lower infusion of volume.

A difficulty faced in this study was the interpretation of steps in the pressure versus volume graph (Figure 1). Maybe the step is related to a small disruption in the tissue or due to needle movements. In such cases, we decided to exclude the graphs from the analysis.

The imbalance between osmotic pressure and hydrostatic pressure in the nasal mucosa from NP patients could contribute to NP formation and it potentially allows us to envision a new treatment strategy. Perhaps not only anti-inflammatory or immunomodulatory drugs, but also means to increase mucosal resistance to prevent hypertropic growth could be used to treat NP. More experiments will be necessary with a larger sample and more accurate method to confirm our results.

\section{Acknowledgement}

We thank Rosana Lima from Surgicalline for the instructions on how to handle and interpret CDS equipment.

This work was supported by a grant to Rogério Pezato, from Conselho Nacional de Pesquisa CNPq.

\section{Authorship contribution}

RP developed the design, collected data, analyzed the data, and drafted the manuscript.

TFPB, RLV and LCG collected data and revised the manuscript. ACS drafted the manuscript and revised it for important intellectual content.

CP, LCG analyzed and revised it for important intellectual content.

All authors gave final approval of the version to be published.

\section{Conflict of interest}

None of the authors report any conflict of interest.

\section{References}

1. Hastan D, Fokkens WJ, Bachert C, et al. Chronic rhinosinusitis in Europe - an underestimated disease. A GA²LEN study. Allergy. 2011; 66: 1216-1223.

2. Fokkens WJ, van Drunen C, Georgalas C, Ebbens F. Role of fungi in pathogenesis of chronic rhinosinusitis: the hypothesis rejected. Curr Opin Otolaryngol Head Neck Surg. 2012; 20: 19-23.

3. Pezato R, Świerczyńska-Krępa M, Niżankowska-Mogilnicka E, Derycke L, Bachert C, Pérez-Novo CA. Role of imbalance of eicosanoid pathways and staphylococcal superantigens in chronic rhinosinusitis. Allergy. 2012; 67: 1347-1356.
4. Hamilos DL. Chronic rhinosinusitis: epidemiology and medical management. Allergy Clin Immunol. 2011; 128: 693-709.

5. Zhang N, Van Zele T, Perez-Novo C, et al Different types of T-effector cells orchestrate mucosal inflammation in chronic sinus disease. J Allergy Clin Immunol. 2008; 122: 961-968 
6. Szczeklik A, Stevenson DD. Aspirin-induced asthma: advances in pathogenesis, diagnosis, and management. J Allergy Clin Immunol. 2003; 111: 913-921.

7. Bachert C, Gevaert P, Holtappels G, Cuvelier C, Van CP. Nasal polyposis: from cytokines to growth. Am J Rhinol 2000; 14: 279-290.

8. Van Bruaene N, Derycke L, Perez-Novo CA, et al. TGF-beta signaling and collagen deposition in chronic rhinosinusitis. J Allergy Clin Immunol. 2009; 124: 253-259.

9. Balsalobre L, Pezato R, Perez-Novo C, et al. Epithelium and stroma from nasal polyp mucosa exhibits inverse expression of TGF$\beta 1$ as compared with healthy nasal mucosa. J Otolaryngol Head Neck Surg. 2013; 42: 29.

10. Pezato R, Voegels RL. Why do we not find polyps in the lungs? Bronchial mucosa as a model in the treatment of polyposis. Med Hypotheses. 2012; 78: 468-470.

11. Heldin $\mathrm{CH}$, Rubin $\mathrm{K}$, Pietras K, Ostman A High interstitial fluid pressure - an obstacle in cancer therapy. Nat Rev Cancer. 2004; 4 806-813.

12. Fokkens WJ, Lund VJ, Mullol J, et al. European Position Paper on Rhinosinusitis and Nasal Polyps 2012. Rhinol Suppl. 2012; (23): 1-298.

13. Torosean S, Flynn B, Axelsson J, et al. Nanoparticle uptake in tumors is mediated by the interplay of vascular and collagen density with interstitial pressure. Nanomedicine. 2013; 9: 151-158.

14. 14. Shieh AC. Biomechanical forces shape the tumor microenvironment. Ann Biomed Eng. 2011; 39: 1379-1389.

Rogério Pezato

Department of Otolaryngology

Head and Neck Surgery

Federal University of São Paulo

Rua dos Otonis

700- piso superior

Vila Clementino

São Paulo

Brazil, CEP 04025002

Tel: +55-11-5575 2552

E-mail: pezatobau@ig.com.br

\section{ADVERTISEMENT}

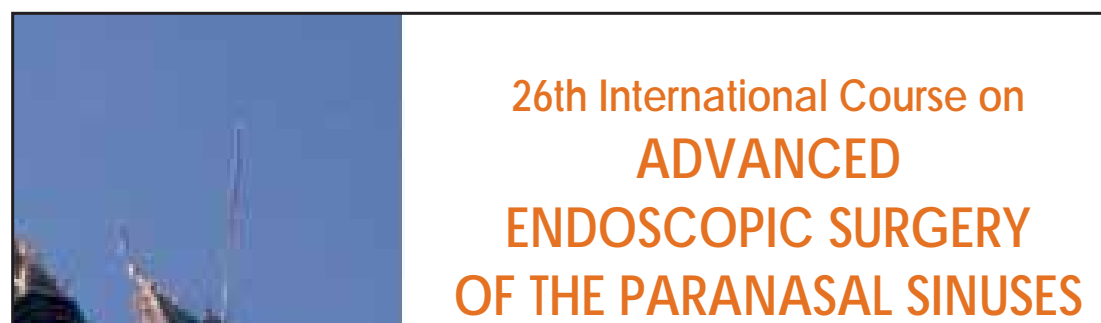

$\mathcal{\&}$

SKULBASE

Ghent (Belgium)

27-30 August 2014

A four-day course in a historical city in Belgium

\section{www.FESS-COURSE.be}

\section{EXIENDED INIERNATIONAL FACULTY}

Bachert C (Ghent), Bemal Sprekelsen M (Barcelona), Gevaert P (Ghent), Hosemann W (Greifswald),Marple

$B$ (Dallas), Schaefer S (New York), Stjä me P

(Stockholm), Van Zele T(G hent), Znreich J (Baltimore)

\section{THE COURSE FEATURES}

\section{1h fully equipped cadaver dissection}

Lectures and round tables on endoscopic sinus surgery and its extensions (DCR, skull base, tumours etc), video sessions, cadaver head demonstration of current techniques, two hands-on cadaver dissections, surgery with navigation, post-operative care, up-date on sinusitis pathophysiology and skull base surgery, interactive discussion with the faculty members. Advanced techniques will be demonstrated. Participants can actively perform surgery on 2 cadaver heads, with full video and mic rodebrider equipment.

\section{REGISTIRATION FE BEFOREJ UNE 1T 2013}

ENT specialist

ENT resident in training* $250 €$

Lectures only

Accompanying person

*reduced registration fee after receipt certific ate

Registration fees may increase after $1^{\text {st }}$ of J une 2014

INFORMATION

University Hospital Ghent, Dept of ENT, HEN Surgeny,

Prof. C. BachertC/o Mrs. Lieve Ectors KorteMeer 16, B- 9000 Ghent

E-mail: Fess@semico.beor fax +32 (0)9 2338597

\section{Under ERS Auspices}

